\title{
RUBBER FRICTION IN AQUEOUS SOLUTIONS CONTAINING IONS
}

\author{
T. P. MORTIMER and K. C. LUDEMA \\ College of Engineering, University of Michigan, Ann. Arbor, Mich. 48104 (U.S.A.) \\ (Received November 6, 1973)
}

SUMMARY

Lubricants ordinarily operate by keeping two sliding surfaces separated. The efficiency of a lubricant may be expressed by equations of hydrodynamics where the mechanical properties of the films of lubricants are adequately defined by the properties of bulk lubricant.

In the case of water lubrication of black rubber at slow speeds and low pressures the lubricating ability of water is shown to be enhanced by addition of electrolytes to the water. It is thought that negative ions from the electrolytic solution collect on each sliding surface, repel each other and prevent the close approach of two sliding surfaces. Thus a thicker water film exists between the sliding surfaces than if the ion layers did not exist. The thicker film results in a reduced viscous drag force, lower than can be accounted for by conventional hydrodynamics.

\section{INTRODUCTION}

Water is an effective lubricant for rubber and under the proper conditions electrolytes may enhance the lubricating ability of water. The effect of the electrolytes may be considerable for some rubbers as shown schematically in Fig. 1. In this preliminary experiment the coefficient of friction between a glass sphere and rubber immersed in water was measured continuously for $10 \mathrm{~min}$ after which a concentrated solution of $\mathrm{NaOH}$ was added to the water. In some cases a large effect is seen. Two types of rubber were used, a black (carbon filled) rubber and a pure natural rubber described as surgical rubber. Five specimens were made from these rubbers. Membranes of each rubber were stretched over the end of a tube and the glass sphere contacted the rubber at the mid region of the span. This produced a low contact pressure. Also a solid body of black rubber was used, and finally membranes of each rubber were stretched over a solid body of black rubber as a backing. The latter was done to separate the effects of contact pressure from the different mode of manufacture of sheet rubber and slab rubber. Figure 1 shows that a large effect was seen when $\mathrm{NaOH}$ was used with black rubber but no effect was seen in the case of the pure rubber. 

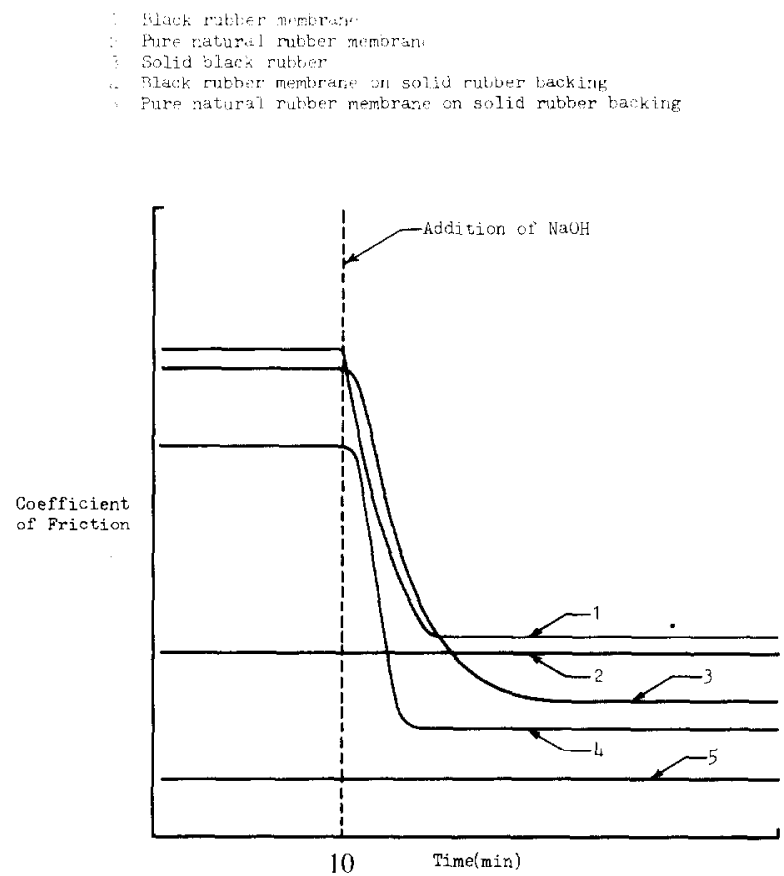

Fig. 1. Effect of electrolyte $\mathrm{NaOH}$ on wet rubber friction.

This paper reports a detailed study of the effect of electrolytes of various kinds on the wet friction of black rubber without attempting to answer the question of why no effect was seen when using pure rubber. Here the term wet rubber friction is used even though viscous drag is the predominant mechanism of friction in these experiments.

In this study it was found that electrolytes in water influence wet rubber friction in at least three ways. One of the ways is by chemically attacking and roughening one or both sliding surfaces. A second way is by changing the physical properties of the solution. For example, the bulk viscosity of the solution is changed by the addition of salts. Figure 2 shows for a number of salts and the base, $\mathrm{NaOH}$, which are collectively known as electrolytes, the ratio of the bulk viscosity of the elcctrolytc solution (i.e., water plus ions) relative to pure water, $\eta / \eta_{0}$. as a function of the molal electrolyte concentration, $G$, at $77^{\circ} \mathrm{F}^{1}$. The change in the bulk viscosity affects both elastohydrodynamic lift and viscous shear forces.

A third way in which salts in water can affect friction is by the adsorption of ions from solution onto the sliding surfaces. Where ions of like charge adsorb on mating sliding surfaces there will be a net electrostatic repulsive force that tends to increase the separation between the surfaces, thereby reducing the viscous drag forces. This is called the electrical double layer effect.

Force interactions between electrical double layers have been studied extensively in the field of colloid stability ${ }^{2}$. In stable colloid solutions the colloid particles are kept from coalescing by double layer repulsive forces between the surfaces of the colloid particles. Roberts and Tabor $^{3}$ investigated the double layer repulsive force interactions between a soft rubber hemisphere and a glass slider 
by measuring the equilibrium electrolyte solution film thickness between the two when they were pressed together. It was possible to deduce the magnitude of the net double layer repulsive force by assuming that it was equal to the sum of the van der Waals force of attraction and the applied normal load. With an applied contact pressure of 2.1 p.s.i. at the center, in distilled water the equilibrium film thickness was "zero" and in $0.01 \mathrm{KCl}$ solution it was $80 \pm 10 \AA\left(1 \AA=10^{-8} \mathrm{~cm}\right)$. When the glass surface was set in motion, the initial force required to shear the equilibrium film was approximately $50 \%$ larger in distilled water than in $\mathrm{KCl}$ solution. McCutchen and Wilkins ${ }^{4}$ investigated the effects of salts in the mucin lubrication of animal joints. In their experiments they simulated an animal joint with a surgical rubber and glass bearing. They found that at a normal contact pressure of about 4.4 p.s.i. a mucin solution of very low ionic strength gave a much higher value of friction than a mucin solution of physiological ionic strength i.e., the ionic strength level of the synovial fluid which lubricates animal joints. The decrease in friction with salts was thought to be due to the force interactions of double layer adsorbed on both the long chain mucin molecules and on the sliding surfaces. McCutchen and Wilkins did not report friction values for distilled water solutions without synovial mucin.

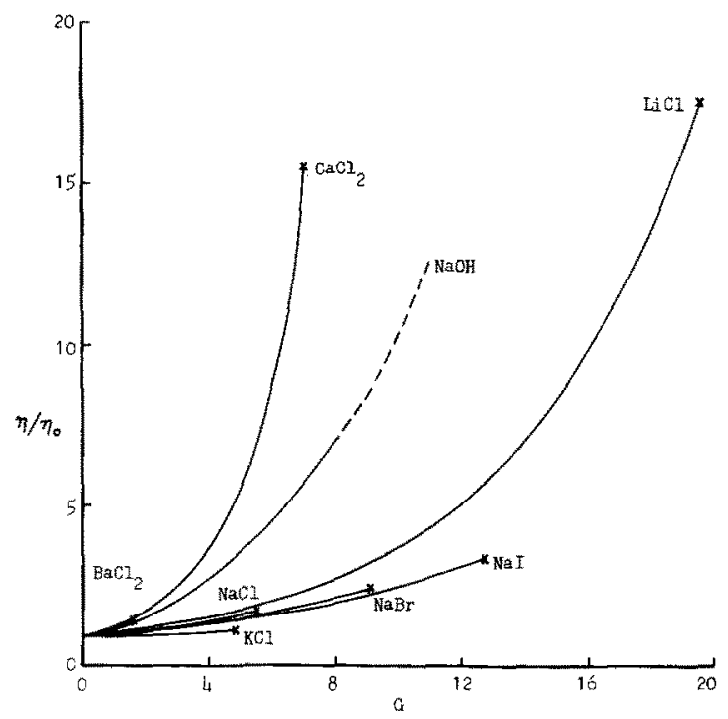

Fig. 2. Effect of electrolytes on bulk viscosity.

The works discussed above do not explore the effects of simple salt solutions on steady state friction. The objective of the present study which is the subject of this paper was to determine to what extent ions in simple electrolytic solutions can affect steady state friction of black rubber and to determine by what mechanism. The major part of the study was done using carbon filled styrene-butadiene rubber of the composition used in some rubber seals, rubber O-rings and automobile tires (see Table I). 
TABLE 1

\begin{tabular}{|c|c|c|}
\hline Ingredient & Parts & Function(s) \\
\hline$\quad \cdots \ldots \ldots$ & & $\begin{array}{llllll}\cdots & \ldots & \ldots & \ldots & \ldots & \ldots\end{array}$ \\
\hline Royalene 505 (SBR) & 100.00 & Main structure \\
\hline $\begin{array}{l}\text { SRF (semi-reinforced } \\
\text { furnace carbon black) }\end{array}$ & 40.00 & Filler: pigment \\
\hline $\begin{array}{l}\text { FEF (fast-extruding } \\
\text { furnace carbon black) }\end{array}$ & 60.00 & Filler; pigment \\
\hline $\mathrm{ZnO}$ & 5,00 & Curing agent \\
\hline Napthenic oil & 100.00 & Physical plasticizer \\
\hline Stearic acid & 1.00 & Improves accelerators \\
\hline Master Batch & 306.00 & \\
\hline Santocure N & 0.80 & Accelerator; mild chemical plasticizer \\
\hline DPG (diphenylguanidine) & 0.30 & Accelerator \\
\hline Sulfur & 1.25 & Curing agent \\
\hline Total & 308.35 & \\
\hline
\end{tabular}

\section{THE EXPERIMENT}

A schematic diagram of the experimental device used for this work is shown in Fig. 3. It consists of a fire polished pyrex sphere which was rotated against a smooth rubber specimen. The axis of rotation of the sphere is perpendicular to the rubber surface. The rubber surface was made smooth by molding against laboratory grade microscope slides. The frictional drag in the contact area applied a torque to the rubber specimen holder which was instrumented so that the frictional torque could be recorded.

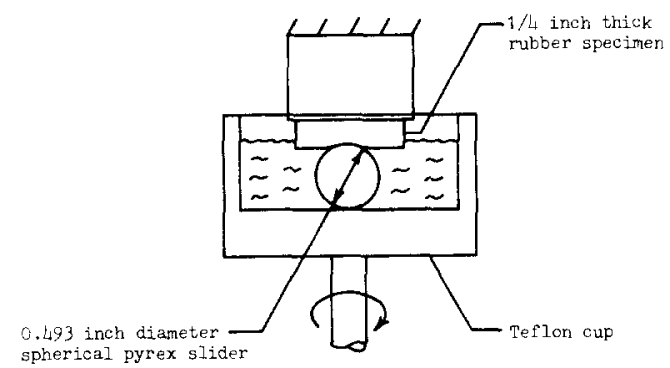

Fig. 3. Schematic or experimental apparatus.

The tests were conducted in the following manner. Sufficient distilled water was placed in the Teflon cup to cover the pyrex sphere. The rubber specimen was then pressed against the pyrex sphere with a dead load. For several minutes after initial contact the rubber settled downward due to the visco-elastic nature of the rubber. An equilibrium in the indentation was achieved in about $10 \mathrm{~min}$ and an avcrage normal contact pressure could be calculated. When this equilibrium was reached the pyrex sphere was set into rotation. After $10 \mathrm{~min}$ of rotation an 
equilibrium value of frictional torque, $T_{1}$, was recorded. Thereafter, a small volume of either more distilled water or electrolyte solution was added to the distilled water already in the Teflon cup. The concentration of the added electrolyte solution was selected to achieve the desired average molal concentration of solution in the Teflon cup. Over the next $13 \mathrm{~min}$ the added ions diffuse into the contact region and a new equilibrium value of frictional torque, $T_{2}$, is recorded. The effect of the added solution on the equilibrium values of frictional torque can be expressed as a percentage change:

$$
\Delta T=\frac{T_{2}-T_{1}}{T_{1}} \times 100
$$

The electrolyte solutions studied were various concentrations of $\mathrm{NaOH}$, $\mathrm{NaCl}, \mathrm{NaBr}, \mathrm{NaI}, \mathrm{LiCl}, \mathrm{CaCl}_{2}$ and $\mathrm{BaCl}_{2}$. The average molal concentrations of the solutions in the Teflon cup after electrolyte additions were $10^{-3}, 10^{-2}, 10^{-1}$, and because of the higher solubility of $\mathrm{NaOH}$ and $\mathrm{LiCl}$, an additional concentration of 1.0 was used. Each electrolyte solution addition test was repeated 3 times, each with a new rubber specimen. The distilled water addition tests were repeated 16 times with 16 new specimens to establish a statistical reference against which the electrolyte solution addition tests were compared. Values of $\Delta T$ from the distilled water addition tests were used in place of electrolyte concentrations of $10^{-5}$ molal on all of the data shown in the Figs. 5 through 12 .

DATA

The recorded data are equilibrium values of $T_{1}$ for distilled water as the lubricant, and $T_{2}$ for the various electrolyte and added distilled water solutions. The values of $T_{1}$ and $T_{2}$ were then used to calculate $\Delta T$. The 3 values of $\Delta T$ for each electrolyte type and concentration, and the 16 values of $\Delta T$ for the distilled water were compared statistically to determine the confidence level probability that the electrolytes affected friction: In many cases the probability was greater than $90 \%$ and as high as $99.95 \%$. It was therefore concluded that the electrolyte effect is statistically significant. The data in Figs. 4 through 12 show average values of 16 repeated tests in distilled water and 3 repeated tests in electrolytes ${ }^{\star}$. The rubber used in these tests was solid bodies of black rubber ${ }^{\star \star}$.

The data in Fig. 4 are shown to illustrate the influence on friction of the bulk viscosity of the electrolyte solutions, which were determined from Fig. 2. Recall that a negative value of $\Delta T$ indicates a decrease in friction after the addition of solution. Because each electrolyte type influences bulk viscosity differently (see Fig.

* Where the statistical probability that the electrolyte affected friction is greater than $90 \%$, the probability is shown in parentheses adjacent to the data point. Note that the data variability as well as the average affect the probability. Tests in distilled water with new rubber specimens before and after each electrolyte addition test series showed no significant change in friction characteristics. In all tests the average contact pressure was 29 p.s.i., the rotational speed of the glass sphere was 4 r.p.m., and the temperature was controlled to $77^{\circ} \pm 2^{\circ} \mathrm{F}$ so the temperature effects on solution viscosity were negligible.

* Royalene 505 SBR rubber supplied by courlesy of Dr. David Sapper at The Uniroyal Tire Company of Detroit, Michigan. See Table I for composition. 


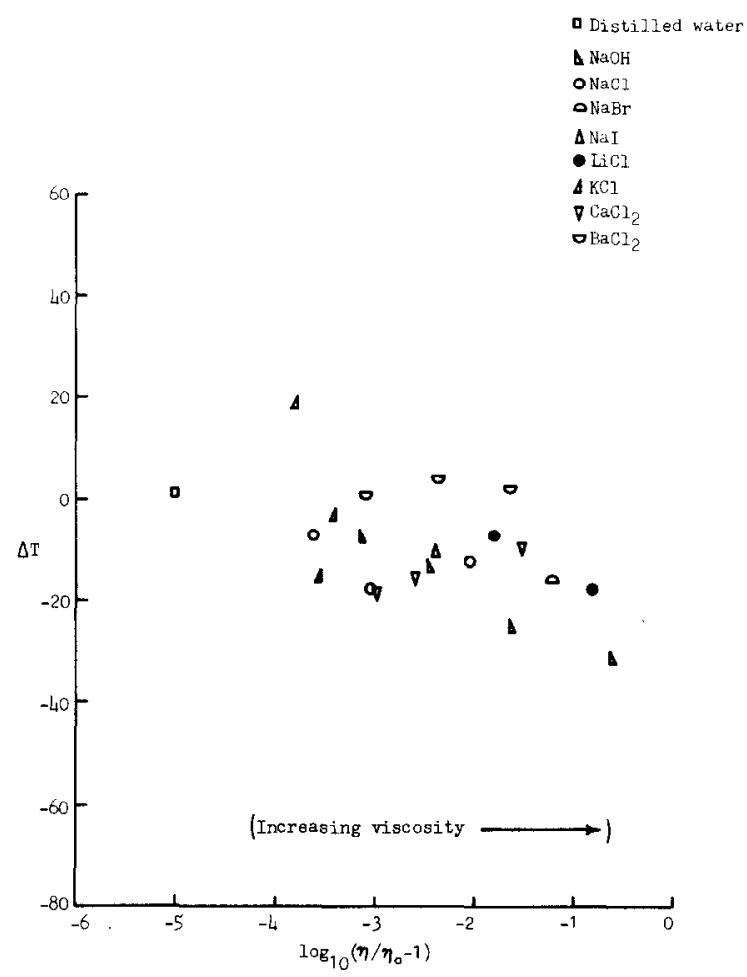

Fig. 4. Effect of bulk viscosity on frictional torque.

2), the values of $\Delta T$ are plotted against viscosity rather than concentration of the electrolyte solution.

Figures 5 through 12 show $\Delta T$ plotted as a function of molal concentration for each electrolyte type. In these figures the shapes and slopes of the curves are the significant features and are discussed below.

\section{DISCUSSION}

This work began with the intention of learning the extent to which electrical double layers influence wet friction of black rubber. It was for this purpose that the specimen configuration shown in Fig. 2 was chosen. A sphere sliding along a linear path on the rubber would be separated from the rubber by an elastohydrodynamically generated fluid film and this effect may obscure the effect of electrical double layer force interactions. Unfortunately, some elastohydrodynamic lift occurred anyway. Even though the glass and rubber specimen surfaces were nominally smooth, the rubber surface in particular did have a fine scale texture or roughness which tends to produce some elastohydrodynamic lift.

In early experiments with other rubber types the rubber surface texture was strongly affected by exposure to electrolyte solutions and this effect alone changed friction. In the tests reported here the surface texture effect was reduced to an insignificant level by selecting a glass and rubber that resisted chemical attack by 

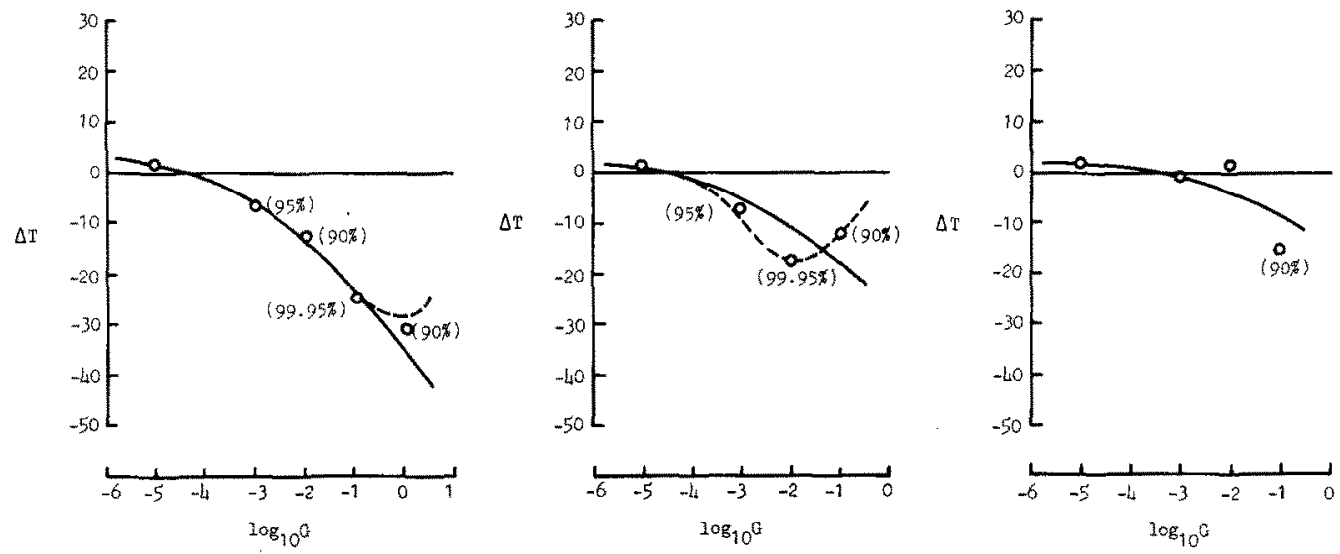

Fig. 5. Effect of $\mathrm{NaOH}$ on frictional torque.

Fig. 6. Effect of $\mathrm{NaCl}$ on frictional torque.

Fig. 7. Effect of $\mathrm{NaBr}$ on frictional torque.
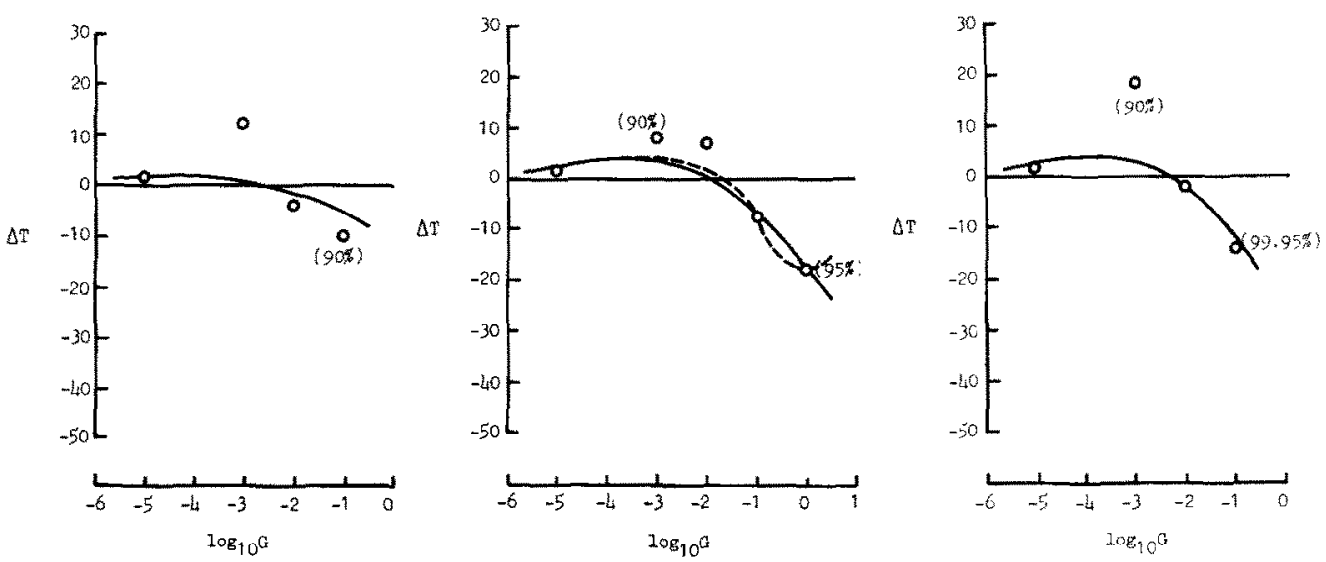

Fig. 8. Effect of NaI on frictional torque.

Fig. 9. Effect of $\mathrm{LiCl}$ on frictional torque.

Fig. 10. Effect of $\mathrm{KCl}$ on frictional torque.

all of the electrolytes used.

Apparently a small elastohydrodynamic lift occurred in most tests as can be seen in Fig. 4. In this plot of $\Delta T$ versus $\log _{10}\left(\eta / \eta_{0}-1\right)$ there is a slight trend toward a reduction in friction with an increase in viscosity, $\eta$, or $\log _{10}\left(\eta / \eta_{0}-1\right)$. The opposite behavior should occur if there were only a simple viscous drag without elastohydrodynamic lift occurring between the glass and rubber. The conclusion from Fig. 4 is that the change in friction in these experiments cannot be explained in terms of viscous effects alone.

Without elastohydrodynamic or double layer effects, with time the film thickness should approach zero and the average coefficient of friction should 

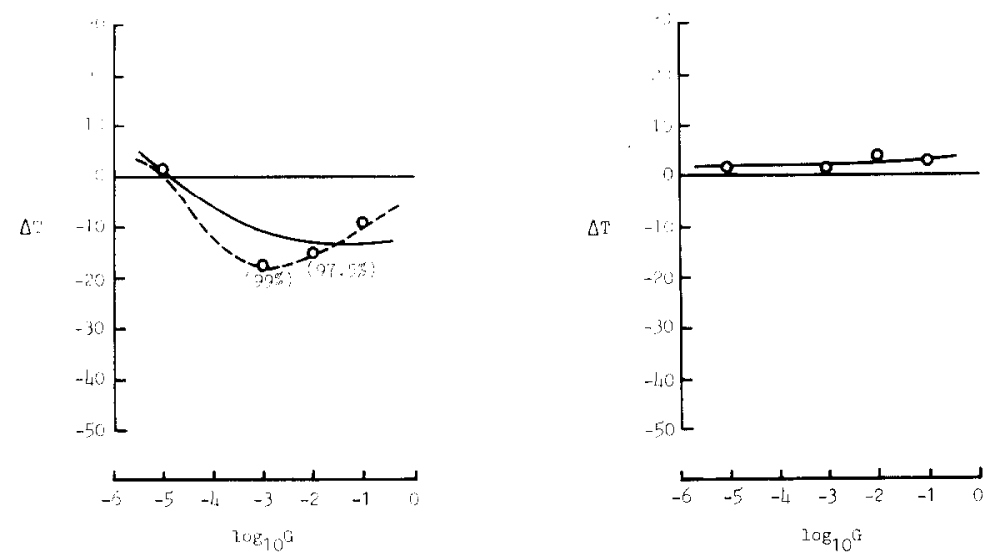

Fig. 11. Effect of $\mathrm{CaCl}_{2}$ on frictional torque.

Fig. 12. Effect of $\mathrm{BaCl}_{2}$ on frictional torque.

approach the "dry" value of about 1.4. In tests, however, the a verage coefficient of friction in distilled water was only about 0.4 . This observation and the absence of severe surface damage of the rubber surface indicates that a fluid film remained in the contact area between the glass and rubber surfaces.

Figures 5 through 12 are plots of the average $\Delta T$ values versus average molal electrolyte concentration, $G$. Collectively, these graphs show trends in friction which are in good agreement with the behavior of electrical double layers interactions. The essential features of electrical double layers for the purpose of this discussion will now be described. Electrolytes in solution dissociate into positive and negative ions. When the solvent is water, the unhydrated negative ions (i.e., anions) may adsorb onto the sliding surfaces. Each anion type is unique in the degree to which it adsorbs and therefore unique in the surface electrical potential that it produces. This is illustrated in Fig. 13 which shows the surface electrical potentials for anion types $a, b$ and $c$. The anion producing the greatest surface electrical potential, $a$ in this case, gives the greatest net double layer interaction force.

Within the bulk of the liquid electrolyte solution, from which some anions adsorb to the solid surface, there is a distribution of anions which have not adsorbed,

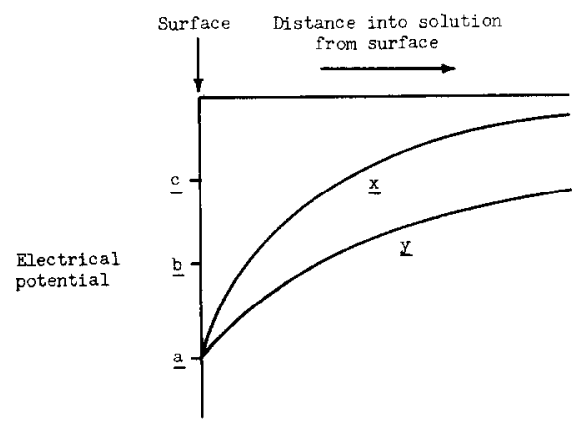

Fig. 13. Electrical potential distribution of double layers. 
in addition to cations. The cations are called counter-ions because the sign of their charge is opposite to that of the adsorbed ions. The cations are electrostatically attracted to the adsorbed anion layer and have the effect of "screening" the surface electrical potential. The result is an electrical potential distribution, two of which are shown in Fig. 13. The counter-ion can be seen to reduce the net effective double layer interaction force as distance from the anion layer increases. The extent of the screening depends upon the cation type (i.e., size, valency, etc.) and the concentration. (Note that the surface potential does not depend primarily upon electrolyte concentration.) The screening effects of different cations on anion type $a$ are illustrated by the two potential distributions for cations $x$ and $y$. Here the $x$ cations screen the surface potential more than the $y$ cations. Here, the $y$ cation may represent a cation hydration complex which is larger than the $x$ cation complex. Alternatively, the $y$ cation may represent a cation of lower valency than the valency of the $x$ cation.

Returning now to the primary subject of this paper, the surface electrical potential produced by adsorbed anions will generally decrease in the order:

$$
\mathrm{OH}^{-}>\mathrm{Cl}^{-}>\mathrm{Br}^{-}>\mathrm{I}^{-}
$$

This should, therefore, be the order of decreasing repulsive force between sliding surfaces upon which anions adsorb. Note that the above order is the reverse of the size order. From Figs. 5, 6, 7 and 8 where $\mathrm{Na}$ is the common cation, it can be seen that the order of effectiveness in decreasing friction of black rubber on glass follows the same order as above. This agreement of anion order not only offers a partial proof for the existence of electrical double layers on black rubber and glass but also confirms the point that the adsorbed ion is the anion. The adsorption of anions onto glass and rubber surfaces was also in agreement with the experimental findings of Roberts and Tabor ${ }^{3}$ and McCutchen and Wilkins ${ }^{4}$.

The effect of the cation type is two fold. First, the effectiveness of the cation hydration complex size in screening the surface potential as illustrated in Fig. 13 decreases in the order:

$$
\mathrm{K}^{+}>\mathrm{Na}^{+}>\mathrm{Li}^{+} \text {and } \mathrm{Ba}^{++}>\mathrm{Ca}^{++}
$$

The di-valent $\mathrm{Ba}^{++}$and $\mathrm{Ca}^{++}$cations are listed separately because the valency of the counter-ion has a separate effect from the hydration complex size and will be discussed below. Comparison of the mono-valent $\mathrm{Cl}$ electrolytes in Figs. 6, 9 and 10 and the di-valent $\mathrm{Cl}$ electrolytes in Figs. 11 and 12 shows that the effectiveness of the cations in decreasing friction is the reverse of the screening order above except $\mathrm{Na}^{+} \cong \mathrm{Li}^{+}$. This is in agreement with double layer effects because the sercening effectiveness is inversely related to the magnitude of the net double layer repulsive force interaction. Similarly, the effectiveness of the cation valency in screening the surface potential as illustrated in Fig. 13 is in the order:

$$
\text { cation }^{++}>\text {cation }^{+}
$$

Comparison of the mono-valent $\mathrm{Cl}$ electrolytes in Figs. 6, 9 and 10 with the di-valent $\mathrm{Cl}$ electrolytes in Figs. 11 and 12 shows that the divalent cations are less effective in decreasing friction. Again, the experimental results are in agreement with the effects of screening on double layer repulsive forces. 
The effect of cation concentration may be seen in the shape of the curves in Figs. 5 through 12. Electrical double layer interaction theory for similarly charged double layers shows that the net repulsive force first increases with concentration. reaches a maximum, and then decreases. The maximum occurs at different concentrations for each electrolyte type and each distance of separation between the solid surfaces. The practical result for the present purpose is that $\Delta T$ should first decrease with increasing concentration, reach a minimum, and then increase again. A trend such as this could be inferred from Figs. 5, 6, 9 and 11. The difficulty in determining the concentration at which the minimum friction exists is in conducting the number of tests required for obtaining statistically reliable data. The purpose in pointing out the influence of electrolyte concentration on the net double layer repulsive force is to explain the general shapes of the curves in Figs. 5 through 12.

\section{CONCLUSION}

There is little doubt that aqueous solutions of some electrolytes significantly reduce the viscous friction between some black rubbers and glass. The probable reason is that ions form electrical double layers on the glass and rubber surfaces which exert a repulsive force and have the effect of preventing very close approach of the rubber to the glass. Roberts and Tabor were able to measure directly the spacing maintained by the double layer when a particular normal force was applied without sliding. In the present work the spacing may be deduced from values of local viscous friction and the bulk viscosity of the electrolyte solution. Assuming that bulk viscosity is the appropriate value to use in the calculation, values of average film thickness of the order of 2 to $40 \AA$ are found, which are in the range of operation of the potential field from the electrical double layers. The contact pressure used was approximately 29 p.s.i., which was over an order of magnitude higher than that used by Roberts and Tabor. In their work a pressure of 29 p.s.i. would have produced a smaller spacing than they observed. In the present work the additional factor of elastohydrodynamic lift resulted in a greater film thickness than would have occurred otherwise.

The phenomenon described above cannot be taken to be universal for all rubbers, as can be seen from Fig. 1. On the other hand the effect is significant. Practical rubber formulations were used in the work reported here, as well as practical salt concentrations. Thus it should be possible to detect the effect of the electron double layer in some special cases of tires on wet roads, for windshield wipers on wet glass, and for rubber seals on smooth shafts.

\section{REFERENCES}

1 International Critical Tables of Numerical Data, Physics, Chemistry, and Technology, Vol. 5, National Research Council, McGraw-Hill, New York, 1929, pp. 12-19.

2 D. J. Shaw, Introduction to Colloid and Surface Chemistry, Butterworths, London, 1966, pp. $137-152$.

3 A. D. Roberts and D. Tabor, Surface forces: direct measurement of repulsive forces due to electrical double layers on solids, Nature, 219 (1968).

4 C. W. McCutchen and J. F. Wilkins, Salt cffects in mucin lubrication. Trans. ASME, F91 (3) (1969). 